\title{
The boxers' fracture: a prospective study of functional recovery
}

\author{
M. L. PORTER, J. P. HODGKINSON, P. HIRST, \\ M. R. WHARTON \& M. CUNLIFFE \\ The Hand Clinic, Royal Preston Hospital, Preston, England
}

\section{SUMMARY}

The rate of functional recovery following fracture of the fifth metacarpal neck is reported. The recovery of power grip and finger grip strength is complete in mos $\vec{\theta}$ patients by two months. The functional outcome is not significantly related to the degree of angulation at the fracture site.

\section{INTRODUCTION}

Fractures of the fifth metacarpal neck are common injuries and account for $20 \%$ of al hand fractures (Hunter \& Cowen, 1970). Volar angulation of the metacarpal head tend 8 to occur (Smith \& Peimer, 1977) although Lowdon (1986) draws attention to the normaP volar angulation of the fifth metacarpal neck. Arafa et al. (1986) concluded that these fractures can be treated without manipulation, regardless of angulation, and by early mobilization, provided rotational deformity is not present.

This prospective study was designed to monitor the rate of recovery of hand function after fifth metacarpal neck fracture and to determine whether functional recovery was related to the severity of angulation.

\section{PATIENTS AND METHODS}

All patients sustaining a classical, isolated 'boxer's' fracture of the fifth metacarpaN between March and August 1986 and presenting to the Royal Preston Hospital, were included in the study. There were 42 patients, with an age range from 9 to 41 year\$ (average 22.3 years): $40(95 \%)$ were male and $2(5 \%)$ female.

Correspondence: Mr M. L. Porter, Senior Orthopaedic Registrar, Wrightington Hospital, Appley Bridge, Nr! Wigan, Lancashire, England. 
All patients were treated with a volar plaster slab with the wrist in 30 degrees of dorsiflexion. The slab was retained for 3 weeks. A broad arm sling was worn for 24-48 hours, but early finger, elbow and shoulder movements were encouraged.

Patients were assessed in the occupational therapy department by an independent observer (M.C.) who had no details of the severity of angulation or degree of displacement at the fracture site. Power grip and grip strength in the ring and little fingers were measured both in the injured and the normal hands. Results were expressed as a percentage of the normal side. Testing was performed immediately after plaster removal and repeated 2 weeks and 2 months later.

\section{RESULTS}

The metacarpal fracture affected the dominant hand in 34 patients $(81 \%)$. The right hand was involved in 32 patients $(76 \%)$ and the left hand in 10 patients $(24 \%)$. Seventeen patients sustained an 'adolescent type' fracture (i.e. before fusion of the growth plate) and the remaining 25 patients sustained 'adult type' fractures.

No attempt was made to reduce the fracture in any of the patients and the degree of angulation was measured from the initial oblique radiographs of the hand. The results are shown in Fig. 1.

All patients had measurement of grip strength when the plaster slab was removed but only $32(76 \cdot 2 \%)$ returned at 2 weeks and $16(38 \cdot 1 \%)$ at 2 months. The 'adolescent' group of fractures had a fairly uniform radiological pattern and return of function was almost immediate, with few patients having less than $50 \%$ of normal function on plaster removal and all having more than $77 \%$ at 2 weeks (Fig. 2). Those patients in the 'adult' group were less compliant in returning for assessment and rehabilitation was slower. Many of the patients in this group had less than $50 \%$ of normal function immediately after plaster removal but improved considerably by 2 weeks and all had more than $70 \%$ normal function at 2 months. In the 'adolescent' group the degree of angulation did not correlate with functional disability. In the 'adult' group, the 2 patients with the poorest grip strength at 2 months each had more than 30 degrees of angulation at the fracture site, although, again, there was no statistically significant correlation between deformity and function.

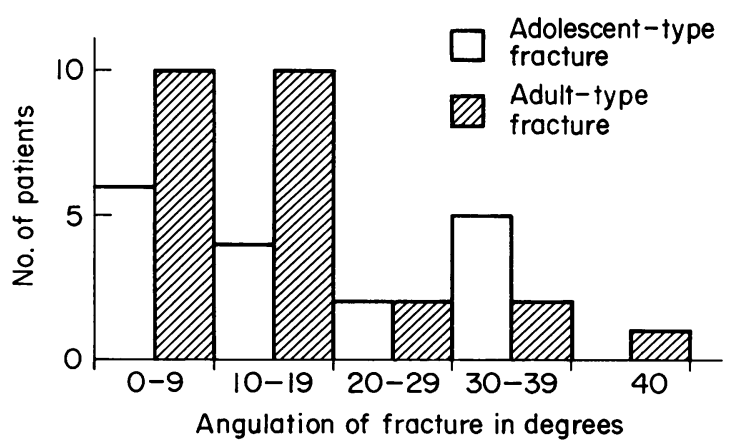

Fig. 1. The histogram shows the degree of angulation at the fracture site, the type of fracture and the number of patients in each group. 


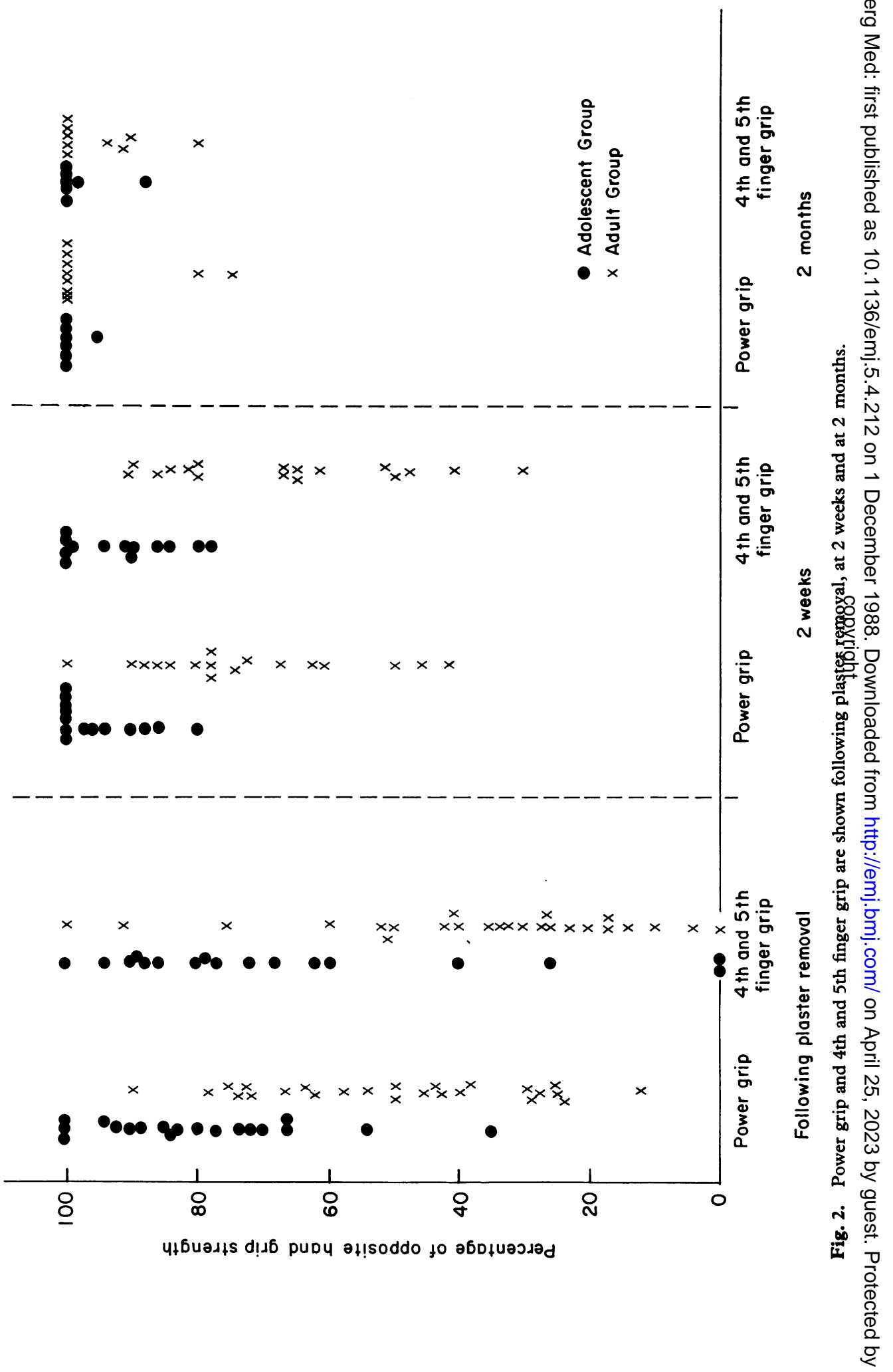




\section{DISCUSSION}

Hunter \& Cowen (1970) showed that considerable angulation of a fifth metacarpal neck fracture was compatible with an early resumption of function and our study confirms that recovery is usually complete 2 months after cast removal.

The rate of functional recovery depended upon whether or not the epiphyseal plate was open; the younger patients recuperating more quickly. Rotational deformity did not appear to be a problem in this series, but we accept that if malrotation is present then it must be corrected as it can be a source of severe disability (Barton, 1984).

We conclude that fractures of the fifth metacarpal neck generally have an excellent prognosis and if rotational deformity is excluded they are best treated with adequate pain relief and early rehabilitation. Surgical intervention or complex methods of splintage are unnecessary and are best avoided.

\section{REFERENCES}

Arafa M., Haines J., Noble J. \& Carden D. (1986) Immediate mobilisation of fractures of the neck of the fifth metacarpal. Injury 17, 277-88.

Barton N. J. (1984) Fractures of the hand. Fournal of Bone and foint Surgery 66-B, 159-67.

Hunter J. M. \& Cowen N. J. (1970) Fifth metacarpal fractures in a compensation clinic population. fournal of Bone and foint Surgery 52-A, 1159-65.

Lowden I. M. R. (1986) Fractures of the metacarpal neck of the little finger. Injury 17, 189-92.

Smith R. J. \& Peimer C. A. (1977) Injuries to the metacarpal bones and joints. Advances in Surgery 2, 341-74. 